\title{
Correction to: Testbeds and Research Infrastructures for the Development of Networks and Communities
}

Honghao Gao, Yuyu Yin, Xiaoxian Yang, and Huaikou Miao

\author{
Correction to: \\ H. Gao et al. (Eds.): \\ Testbeds and Research Infrastructures for the Development \\ of Networks and Communications, LNICST 270, \\ https://doi.org/10.1007/978-3-030-12971-2
}

The original version of the book unfortunately contained a mistake in the book title. The title of the book has been corrected. 